FACTA UNIVERSITATIS (NIŠ)

Ser. Math. Inform. Vol. 33, No 5 (2018), 751-771

https://doi.org/10.22190/FUMI1805751K

\title{
NONLINEAR INVARIANTS OF PLANAR POINT CLOUDS TRANSFORMED BY MATRICES
}

\author{
Stelios Kotsios and Evangelos Melas
}

\begin{abstract}
The goal of this paper is to present invariants of planar point clouds, that is functions which take the same value before and after a linear transformation of a planar point cloud via a $2 \times 2$ invertible matrix. In the approach we adopt here, these invariants are functions of two variables derived from the least squares straight line of the planar point cloud under consideration. A linear transformation of a point cloud induces a nonlinear transformation of these variables. The said invariants are solutions to certain Partial Differential Equations, which are obtained by employing Lie theory. We find cloud invariants in the general case of a four-parameter transformation matrix, as well as, cloud invariants of various one-parameter sets of transformations which can be practically implemented. Case studies and simulations which verify our findings are also provided.
\end{abstract}

\section{Introduction}

Analysing level shapes is the key problem in many computer science areas, as image analysis, geometric computing, optical character recognition e.t.c. $[2,7]$. Usually, by means of the modern sensing technology, we make detailed scans of complex plane objects by generating point cloud data, consisting from thousands or millions of points. Then we study the underlying properties, either by creating appropriate models or by discovering properties which remain constant under sets of transformations or under the influence of noise distortions.

In particular, when we deal with planar set of points, a basic approach, which is widely used, is that of determining quantities which can characterize collectively the behaviour of the whole set, as well as its change, when a transformation is applied to it. In other words, we determine quantities which can represent the planar set of points under consideration, as a whole.

One approach along this line is the classical work of Ming-Kuei Hu [6], who introduced the moment invariants methodology, followed in the course of time by

Received June 23, 2018; accepted October 23, 2018

2010 Mathematics Subject Classification. 35A20, 22E05 ; 35A09, 34A25, 34A30 
many others $[3,4,9,10]$, to mention but a few. The key element of their approach was to introduce the so-called moments of planar figures, in order to identify a planar geometrical figure as a whole, and then to study their invariants under translation, similitude and orthogonal transformations.

In the present paper, we consider planar set of points, called henceforth point cloud or cloud of points. We advocate a different approach, and in order to characterize collectively the behaviour of the whole cloud of points we introduce two variables $M$ and $H$. These variables stem from the least squares line assigned to these points. In fact $M$ is the slope of this line, and $H$ is a variation of the $y$-intercept of this line.

Any transformation of the cloud of points, by means of a $2 \times 2$ matrix, induces a nonlinear transformation, to be precise a rational one, of the quantities $M$ and $H$. We assume throughout this paper that any $2 \times 2$ transformation matrix of a cloud of points is invertible.

The purpose of this paper is to find cloud invariants, or shortly invariants, expressed in terms of the variables $M$ and $H$. By the term "invariants" [8] we mean functions which take the same value at the original and at the transformed values of $M$ and $H$, when a cloud of points undergoes a transformation with a $2 \times 2$ matrix. In order to solve this problem we use Lie Theory $[5,1]$. As a result, the problem is reduced to solving certain Partial Differential Equations. Any solution to these PDEs, provides us with a cloud invariant.

Since the problem, in its general form, does not have a solution which can be practically implemented, we are examining the special case of an one-parameter set of transformations. This is the case when the entries of a transformation matrix are functions of one parameter only. In this case a general solution is found by using Lie theory implemented with symbolic computation.

At first sight it might seem that restricting ourselves to a one-parameter set of transformations, useful as it may be, cannot be of great use. However, this is not the case, because as we point out in section 5.2.1. any given matrix belongs to one such one-parameter set of transformations. As a result we find a family of cloud invariants for any given matrix and this certainly lends itself to practical implementation.

By practical implementation we mean that these invariants can be used as a tool for studying changes of planar figures and for creating proper software which monitors and displays these changes in real time. This would have many applications in optical character recognition, as well as, in image analysis and computer graphics techniques; icons created by the same "source" will be readily identified.

This potential application of our results suggests a direction for future research. The cloud of points may come from an icon which has a parabolic- like shape. In this case it is natural to look for cloud invariants which are expressed in terms of variables which appear as coefficients, or variations thereof, of the parabola which is the best fit for the cloud points we consider. Comparison with already existing methodologies, which address the same questions, via simulations and computational experiments, will be also the subject of future research. 
In section 2. we introduce the variables $M$ and $H$, and we find the transformation of these variables which is induced from a transformation with a $2 \times 2$ matrix of the cloud of points under consideration. In section 3 . we define the notion of an invariant function, and we prove in the current case, Lie's theory fundamental result that the nonlinear invariant condition is equivalent to a linear condition provided that the invariant function is properly analytic. Moreover, by using this linear condition, we find the cloud invariants in the general case of a four-parameter transformation matrix of the cloud of points under consideration. In section 4 . we find a family of cloud invariants for a general one-parameter set of transformations. In section 5 . we find families of cloud invariants for various sets of transformations. We also find a family of cloud invariants for a "linear" one-parameter set of transformations and we point out that any given matrix belongs to such a set. In section 6 . we verify our results with simulations and computational experiments in a cloud of 10.000 points. In section 7 . we close the paper with some concluding remarks.

\section{The Basic Quantities and their Transformations}

In this section we present two quantities $M$ and $H$ which characterize collectively a cloud of points and serve as the independent variables of the invariant functions we are going to construct. They originate from the least squares straight line fitted to the cloud of points under consideration.

Let $\left(x_{i}, y_{i}\right), i=1, \ldots, N$, be a cloud of points on the plane. We define the quantities:

$$
\begin{aligned}
M & =\frac{N \sum_{i=1}^{N} x_{i} y_{i}-\sum_{i=1}^{N} x_{i} \sum_{i=1}^{N} y_{i}}{N \sum_{i=1}^{N} x_{i}^{2}-\left(\sum_{i=1}^{N} x_{i}\right)^{2}}, \text { and }, \\
H & =\frac{N \sum_{i=1}^{N} y_{i}^{2}-\left(\sum_{i=1}^{N} y_{i}\right)^{2}}{N \sum_{i=1}^{N} x_{i}^{2}-\left(\sum_{i=1}^{N} x_{i}\right)^{2}}
\end{aligned}
$$

$M$ is the slope of the least squares straight line and $H$ is suggested by the calculations. We call them the linear coefficients of the cloud. Sometimes $M$ is referred as the slope of the cloud and $H$ as the constant term of the cloud. A transformation of the cloud of points under the action of a $2 \times 2$ matrix induces a transformation to $M$ and $H$. This last transformation is of prime importance to our construction of invariant functions and so we proceed to find it. Firstly, we need a definition.

Definition 2.1. Let $\left(x_{i}, y_{i}\right), i=1, \ldots, N$, be a cloud of points and $A=\left(\begin{array}{ll}\alpha & \beta \\ \gamma & \delta\end{array}\right)$, $\alpha, \beta, \gamma, \delta \in \mathbf{R}$, a given $2 \times 2$ matrix. Let us suppose that every point $\left(x_{i}, y_{i}\right)$, $i=1, \ldots, N$, of the cloud undergoes a transformation $T_{A}:\left(\begin{array}{c}x_{i} \\ y_{i}\end{array}\right) \rightarrow\left(\begin{array}{c}\hat{x}_{i} \\ \hat{y}_{i}\end{array}\right)$ according to the rule $\left(\begin{array}{c}\hat{x}_{i} \\ \hat{y}_{i}\end{array}\right)=A\left(\begin{array}{c}x_{i} \\ y_{i}\end{array}\right)$. 
We say that the cloud is transformed under the matrix $A$, and in particular we say that the cloud $\left(\hat{x}_{i}, \hat{y}_{i}\right), i=1, \ldots, N$, is the transformation of the cloud $\left(x_{i}, y_{i}\right)$, $i=1, \ldots, N$, under the matrix $A$.

The transformation of $M$ and $H$ induced by a transformation of the cloud of points via a matrix $A$ is given in the following Theorem.

Theorem 2.1. Let $\left(x_{i}, y_{i}\right), i=1,2, \ldots, N$, be a cloud of points with linear coefficients $M$ and $H$. Let $\left(\hat{x}_{i}, \hat{y}_{i}\right), i=1,2, \ldots, N$, be the transformation of the cloud $\left(x_{i}, y_{i}\right), i=1, \ldots, N$, under a matrix $A=\left(\begin{array}{ll}\alpha & \beta \\ \gamma & \delta\end{array}\right), \alpha, \beta, \gamma, \delta \in \mathbf{R}$. Let $\hat{M}$ and $\hat{H}$ be the linear coefficients of the cloud $\left(\hat{x}_{i}, \hat{y}_{i}\right), i=1,2, \ldots, N$. Then the following relations hold

$$
\begin{aligned}
\hat{M} & =\frac{(\alpha \delta+\beta \gamma) M+\beta \delta H+\alpha \gamma}{2 \alpha \beta M+\beta^{2} H+\alpha^{2}} \\
\hat{H} & =\frac{2 \gamma \delta M+\delta^{2} H+\gamma^{2}}{2 \alpha \beta M+\beta^{2} H+\alpha^{2}}
\end{aligned}
$$

Proof: Let $\left(x_{i}, y_{i}\right), i=1,2, \ldots, N$, be a cloud of points with linear coefficients $M$ and $H$. It is convenient to define the quantities $M_{n}, H_{n}$, and $D$, as follows

$$
\begin{aligned}
M_{n} & =N \sum_{i=1}^{N} x_{i} y_{i}-\sum_{i=1}^{N} x_{i} \sum_{i=1}^{N} y_{i} \\
H_{n} & =N \sum_{i} y_{i}^{2}-\left(\sum_{i} y_{i}\right)^{2} \\
D & =N \sum_{i} x_{i}^{2}-\left(\sum_{i} x_{i}\right)^{2}
\end{aligned}
$$

The relations (2.1) and (2.2) which define the linear coefficients $M$ and $H$ of the cloud of points under consideration can now be written in the following shorter form:

$$
M=\frac{M_{n}}{D}, \quad H=\frac{H_{n}}{D} .
$$

A transformation of the cloud of points under a matrix $A=\left(\begin{array}{cc}\alpha & \beta \\ \gamma & \delta\end{array}\right), \alpha, \beta, \gamma, \delta \in$ $\mathbf{R}$, induces a transformation to the quantities $M_{n}, H_{n}, D$. The induced transformed values $\hat{M}_{n}, \hat{H}_{n}, \hat{D}$, which are assigned to the cloud $\left(\hat{x}_{i}, \hat{y}_{i}\right), i=1,2, \ldots, N$, are calculated as follows:

$$
\begin{gathered}
\hat{M}_{n}=N \sum_{i} \hat{x}_{i} \hat{y}_{i}-\sum_{i} \hat{x}_{i} \sum_{i} \hat{y}_{i}= \\
=N \sum_{i}\left(\alpha x_{i}+\beta y_{i}\right)\left(\gamma x_{i}+\delta y_{i}\right)-\sum_{i}\left(\alpha x_{i}+\beta y_{i}\right) \sum_{i}\left(\gamma x_{i}+\delta y_{i}\right)=
\end{gathered}
$$




$$
\begin{aligned}
=\alpha \gamma & {\left[N \sum_{i}^{2}-\left(\sum_{i} x_{i}\right)^{2}\right]+(\alpha \delta+\beta \gamma)\left[N \sum_{i} x_{i} y_{i}-\sum_{i} x_{i} \sum_{i} y_{i}\right]+} \\
& +\beta \delta\left[N \sum_{i} y_{i}^{2}-\left(\sum_{i} y_{i}^{2}\right)\right]=\alpha \gamma D+(\alpha \delta+\beta \gamma) M_{n}+\beta \delta H_{n}, \\
\hat{H}_{n}= & N \sum_{i} \hat{y}_{i}^{2}-\left(\sum_{i} \hat{y}_{i}\right)^{2}=N \sum_{i}\left(\gamma x_{i}+\delta y_{i}\right)-\left[\sum_{i}\left(\gamma x_{i}+\delta y_{i}\right)\right]^{2}= \\
& =\gamma^{2}\left[N \sum_{i} x_{i}^{2}-\left(\sum_{i} x_{i}\right)^{2}\right]+\delta^{2}\left[N \sum_{i} y_{i}^{2}-\left(\sum_{i} y_{i}^{2}\right)\right]+ \\
& +2 \gamma \delta\left[N \sum_{i} x_{i} y_{i}-\sum_{i} x_{i} \sum_{i} y_{i}\right]=\gamma^{2} D+\delta^{2} H_{n}+2 \gamma \delta M_{n},
\end{aligned}
$$

and,

$$
\begin{aligned}
\hat{D} & =N \sum_{i} \hat{x}_{i}^{2}-\left(\hat{x}_{i}\right)^{2}=N \sum_{i}\left(\alpha x_{i}+\beta y_{i}\right)^{2}-\left[\sum_{i}\left(\alpha x_{i}+\beta y_{i}\right)\right]^{2}= \\
& =\alpha^{2}\left[N \sum_{i} x_{i}^{2}-\left(\sum_{i} x_{i}\right)^{2}\right]+\beta^{2}\left[N \sum_{i} y_{i}^{2}-\left(\sum_{i} y_{i}^{2}\right)\right]+ \\
& +2 \alpha \beta\left[N \sum_{i} x_{i} y_{i}-\sum_{i} x_{i} \sum_{i} y_{i}\right]=\alpha^{2} D+\beta^{2} H_{n}+2 \alpha \beta M_{n} .
\end{aligned}
$$

From relations $(2.8),(2.9),(2.10)$, and $(2.11)$, we conclude that the linear coefficients $\hat{M}$ and $\hat{H}$ of the cloud $\left(\hat{x}_{i}, \hat{y}_{i}\right), i=1,2, \ldots, N$, are given by the relations $(2.3),(2.4)$ and the theorem has been proved.

We denote the set of transformations $(2.3)$ and $(2.4)$ by $\mathcal{T}(A)$. These are transformations of the form

$$
\hat{M}=\mathcal{M}(M, H, \alpha, \beta, \gamma, \delta), \quad \hat{H}=\mathcal{H}(M, H, \alpha, \beta, \gamma, \delta) .
$$

If $A$ is a matrix with entries $(\alpha, \beta ; \gamma, \delta)$, then we can associate to it an element of the set $\mathcal{T}(A)$, namely the transformation given by (2.3) and (2.4). We denote this transformation by $\mathcal{T}(A)_{(\alpha, \beta, \gamma, \delta)}$. The following remarks are in order regarding this association:

- The set of transformations $\mathcal{T}(A)$ form a Lie group, under the usual composition of transformations, if and only if the set of matrices $A$ form also a Lie group, under the usual multiplication of matrices, namely the group $G L(2)$, i.e., the group of $2 \times 2$ invertible matrices. 
- This association is not one-to-one. Indeed, one can easily check that

$$
\mathcal{T}(A)_{(\alpha, \beta, \gamma, \delta)}=\mathcal{T}(A)_{(\kappa \alpha, \kappa \beta, \kappa \gamma, \kappa \delta)},
$$

$\kappa \in R, \kappa \neq 0$. Therefore all matrices $\kappa A$ are associated to the same element $\mathcal{T}(A)_{(\alpha, \beta, \gamma, \delta)}$ of $\mathcal{T}(A)$.

- We can make the association between the sets $A$ and $\mathcal{T}(A)$ one-to-one by assigning arbitrarily a fixed non-zero value to any of the entries $(\alpha, \beta ; \gamma, \delta)$ of the matrices of the set $A$.

- In this paper we prefer not to make this association one-to-one because this may give the false impression that restrictions are imposed to the set of transformations $A$ which act on the cloud of points under consideration.

- Needless to say that the results are identical regardless of whether we make or we do not make the association between the sets $A$ and $\mathcal{T}(A)$ one-to-one.

We note that in the search for invariants we do not need to restrict to the case where $A$, and therefore $\mathcal{T}(A)$, is a group. As it will become evident from the proof in the next section, and as it will be demonstrated in the example given in subsection 5.2.1., what it is really necessary is that the set $A$, and subsequently the set $\mathcal{T}(A)$, must contain the identity element. The identity elements of both $A$ and $\mathcal{T}(A)$ are obtained when $\alpha=1, \beta=0, \gamma=0$, and, $\delta=1$. For brevity we write $e=(1,0,0,1)$ and we denote by $e_{i}, i=1,2,3,4$, its components.

\section{Invariants}

A main objective in cloud of points theory is that of finding invariants. These are quantities which remain unchanged whenever a cloud of points is transformed under the action of a $2 \times 2$ matrix. Invariant quantities enable us to recognize clouds of points arising from the same "source".

In our approach the entities which identify a cloud of points are $M$ and $H$. Therefore, we are looking for invariants which are functions of these two quantities. This is formalized in the following definition:

Definition 3.1. Let $\left(x_{i}, y_{i}\right), i=1, \ldots, N$, be a cloud of points on the plane with linear coefficients $M$ and $H$. Let $\left(\hat{x}_{i}, \hat{y}_{i}\right), i=1, \ldots, N$, be the transformation of the cloud $\left(x_{i}, y_{i}\right), i=1, \ldots, N$, under a matrix $A$. Let $\hat{M}, \hat{H}$ be the linear coefficients of the cloud $\left(\hat{x}_{i}, \hat{y}_{i}\right), i=1, \ldots, N . \hat{M}$ and $\hat{H}$ are the transformed values of $M$ and $H$ under the induced set of transformations $\mathcal{T}(A)$. We say that a function $I: \mathbf{R}^{2} \rightarrow \mathbf{R}$ is a cloud invariant if and only if

$$
I(\hat{M}, \hat{H})=I(M, H) .
$$


The next theorem is the key result in our study because it provides us with a mechanism for finding cloud invariants. Its proof, is the proof in our case, of Lie's theory fundamental result [1] that the nonlinear condition (3.1) is equivalent to a linear condition provided that the invariant function is properly analytic. The details are as follows:

Theorem 3.1. Let $\left(x_{i}, y_{i}\right), i=1, \ldots, N$, be a cloud of points on the plane with linear coefficients $M$ and $H$, and let $\left(\hat{x}_{i}, \hat{y}_{i}\right), i=1, \ldots, N$, be the transformation of the cloud $\left(x_{i}, y_{i}\right), i=1, \ldots, N$, under a matrix $A=\left(\begin{array}{cc}\alpha & \beta \\ \gamma & \delta\end{array}\right)$. A function $I: \mathbf{R}^{2} \rightarrow \mathbf{R}$, analytic in the parameters $\alpha, \beta, \gamma, \delta$, is a cloud invariant if and only if the following equations hold simultaneously

$$
\begin{aligned}
& \xi_{\alpha}^{1} \frac{\partial I}{\partial M}+\xi_{\alpha}^{2} \frac{\partial I}{\partial H}=0, \\
& \xi_{\beta}^{1} \frac{\partial I}{\partial M}+\xi_{\beta}^{2} \frac{\partial I}{\partial H}=0, \\
& \xi_{\gamma}^{1} \frac{\partial I}{\partial M}+\xi_{\gamma}^{2} \frac{\partial I}{\partial H}=0, \\
& \xi_{\delta}^{1} \frac{\partial I}{\partial M}+\xi_{\delta}^{2} \frac{\partial I}{\partial H}=0,
\end{aligned}
$$

where,

$$
\xi_{Q}^{1}=\left(\frac{\partial \hat{M}}{\partial Q}\right)_{e}=\frac{\partial \mathcal{M}(M, H, 1,0,0,1)}{\partial Q}, \xi_{Q}^{2}=\left(\frac{\partial \hat{H}}{\partial Q}\right)_{e}=\frac{\partial \mathcal{H}(M, H, 1,0,0,1)}{\partial Q}
$$

$Q=\alpha, \beta, \gamma, \delta$

Proof: Let $\left(x_{i}, y_{i}\right), i=1, \ldots, N$, be a cloud of points on the plane with linear coefficients $M$ and $H$. Let $\left(\hat{x}_{i}, \hat{y}_{i}\right), i=1, \ldots, N$, be the transformation of the cloud $\left(x_{i}, y_{i}\right), i=1, \ldots, N$, under a matrix $A=\left(\begin{array}{cc}\alpha & \beta \\ \gamma & \delta\end{array}\right)$. Let $\hat{M}, \hat{H}$ be the linear coefficients of the cloud $\left(\hat{x}_{i}, \hat{y}_{i}\right), i=1, \ldots, N . \hat{M}$ and $\hat{H}$ are the transformed values of $M$ and $H$ under the induced set of transformations $\mathcal{T}(A)$, given by equations (2.3) and (2.4). Let $I(\hat{M}, \hat{H})$ be a real-valued function analytic in the parameters $\alpha, \beta, \gamma$, and $\delta$. The Taylor expansion of $I(\hat{M}, \hat{H})$, with center $e$, reads:

$$
\begin{aligned}
I(\hat{M}, \hat{H})= & I(M, H)+(\alpha-1)\left(\frac{\partial I(\hat{M}, \hat{H})}{\partial \alpha}\right)_{e}+\beta\left(\frac{\partial I(\hat{M}, \hat{H})}{\partial \beta}\right)_{e}+ \\
& \gamma\left(\frac{\partial I(\hat{M}, \hat{H})}{\partial \gamma}\right)_{e}+(\delta-1)\left(\frac{\partial I(\hat{M}, \hat{H})}{\partial \delta}\right)_{e}++\frac{1}{2 !}\left((\alpha-1)^{2}\right. \\
& \left(\frac{\partial^{2} I(\hat{M}, \hat{H})}{\partial \alpha^{2}}\right)_{e}+\beta^{2}\left(\frac{\partial^{2} I(\hat{M}, \hat{H})}{\partial \beta^{2}}\right)_{e}+\gamma^{2}\left(\frac{\partial^{2} I(\hat{M}, \hat{H})}{\partial \gamma^{2}}\right)_{e}+
\end{aligned}
$$




$$
\begin{aligned}
& (\delta-1)^{2}\left(\frac{\partial^{2} I(\hat{M}, \hat{H})}{\partial \delta^{2}}\right)_{e}+2(\alpha-1) \beta\left(\frac{\partial I(\hat{M}, \hat{H})}{\partial \alpha}\right)_{e}+\cdots \\
& \left.2 \gamma(\delta-1)\left(\frac{\partial I(\hat{M}, \hat{H})}{\partial \gamma}\right)_{e}\left(\frac{\partial I(\hat{M}, \hat{H})}{\partial \delta}\right)_{e}\right)+\cdots
\end{aligned}
$$

The form of the functional dependence of $I(\hat{M}, \hat{H})$ on the parameters $\alpha, \beta, \gamma, \delta$ allows to simplify (3.6) in a way which suggests the conclusion of the theorem. To illustrate the point at hand we use the chain rule to evaluate the derivative $\left(\frac{\partial I(\hat{M}, \hat{H})}{\partial \alpha}\right)_{e}$ :

$$
\left(\frac{\partial I(\hat{M}, \hat{H})}{\partial \alpha}\right)_{e}=\left(\frac{\partial I(\hat{M}, \hat{H})}{\partial \hat{M}} \frac{\partial \hat{M}}{\partial \alpha}+\frac{\partial I(\hat{M}, \hat{H})}{\partial \hat{H}} \frac{\partial \hat{H}}{\partial \alpha}\right)_{e}
$$

(3.8) $\left(\frac{\partial I(\hat{M}, \hat{H})}{\partial \alpha}\right)_{e}=\left(\frac{\partial I(\hat{M}, \hat{H})}{\partial \hat{M}}\right)_{e}\left(\frac{\partial \hat{M}}{\partial \alpha}\right)_{e}+\left(\frac{\partial I(\hat{M}, \hat{H})}{\partial \hat{H}}\right)_{e}\left(\frac{\partial \hat{H}}{\partial \alpha}\right)_{e}$

By introducing the quantities:

$$
\xi_{Q}^{1}=\left(\frac{\partial \hat{M}}{\partial Q}\right)_{e}, \xi_{Q}^{2}=\left(\frac{\partial \hat{H}}{\partial Q}\right)_{e}, Q=\alpha, \beta, \gamma, \delta
$$

and by noting

$$
\left(\frac{\partial I(\hat{M}, \hat{H})}{\partial \hat{M}}\right)_{e}=\frac{\partial I(M, H)}{\partial M}, \text { and, }\left(\frac{\partial I(\hat{M}, \hat{H})}{\partial \hat{H}}\right)_{e}=\frac{\partial I(M, H)}{\partial H}
$$

we can rewrite equation (3.8) in the following shorter form

$$
\left(\frac{\partial I(\hat{M}, \hat{H})}{\partial \alpha}\right)_{e}=\xi_{\alpha}^{1} \frac{\partial I(M, H)}{\partial M}+\xi_{\alpha}^{2} \frac{\partial I(M, H)}{\partial H}
$$

By introducing the operator

$$
X_{\alpha}=\xi_{\alpha}^{1} \frac{\partial}{\partial M}+\xi_{\alpha}^{2} \frac{\partial}{\partial H},
$$

we rewrite equation (3.11) as

$$
\left(\frac{\partial I(\hat{M}, \hat{H})}{\partial \alpha}\right)_{e}=X_{\alpha} I
$$


where for short we wrote $I$ instead of $I(M, H)$. For the second order derivative $\left(\frac{\partial^{2} I(\hat{M}, \hat{H})}{\partial \alpha^{2}}\right)_{e}$ we have:

$$
\begin{aligned}
\left(\frac{\partial^{2} I(\hat{M}, \hat{H})}{\partial \alpha^{2}}\right)_{e} & =\left(\frac{\partial\left(\frac{\partial I(\hat{M}, \hat{H})}{\partial \alpha}\right)}{\partial \alpha}\right)_{e}=\xi_{\alpha}^{1} \frac{\partial\left(\frac{\partial I(\hat{M}, \hat{H})}{\partial \alpha}\right)_{e}}{\partial M}+\xi_{\alpha}^{2} \frac{\partial\left(\frac{\partial I(\hat{M}, \hat{H})}{\partial \alpha}\right)_{e}}{\partial H} \\
& =X_{\alpha}\left(X_{\alpha} I\right)
\end{aligned}
$$

A similar analysis applies to the derivatives of all orders in the Taylor expansion (3.6). As a result the Taylor expansion (3.6) reads:

$$
\begin{aligned}
& I(\hat{M}, \hat{H})=I(M, H)+\sum_{i=1}^{4}\left(\mathcal{Q}_{i}-e_{i}\right)\left(X_{\mathcal{Q}_{i}} I\right)+ \\
& \quad \frac{1}{2 !} \sum_{i, j=1}^{4}\left(\mathcal{Q}_{i}-e_{i}\right)\left(\mathcal{Q}_{j}-e_{j}\right) X_{\mathcal{Q}_{i}}\left(X_{\mathcal{Q}_{j}} I\right)+ \\
& \quad \frac{1}{3 !} \sum_{i, j, k=1}^{4}\left(\mathcal{Q}_{i}-e_{i}\right)\left(\mathcal{Q}_{j}-e_{j}\right)\left(\mathcal{Q}_{k}-e_{k}\right) X_{\mathcal{Q}_{i}}\left(X_{\mathcal{Q}_{j}}\left(X_{\mathcal{Q}_{k}} I\right)\right)+\cdots
\end{aligned}
$$

For convenience, by $\mathcal{Q}$ we denote the vector $(\alpha, \beta, \gamma, \delta)$, and by $\mathcal{Q}_{i}, i=1,2,3,4$, its components.

From equation (3.15) we conclude that when the linear infinitesimal conditions

$$
X_{\mathcal{Q}_{i}} I=0, \quad \mathcal{Q}_{i}=\alpha, \beta, \gamma, \delta,
$$

are satisfied then $I(\hat{M}, \hat{H})=I(M, H)$. Therefore $I$ is a cloud invariant.

Conversely, when $I$ is a cloud invariant, then $I(\hat{M}, \hat{H})=I(M, H)$, and equation (3.15) gives:

$$
\begin{gathered}
\sum_{i=1}^{4}\left(\mathcal{Q}_{i}-e_{i}\right)\left(X_{\mathcal{Q}_{i}} I\right)+\frac{1}{2 !} \sum_{i, j=1}^{4}\left(\mathcal{Q}_{i}-e_{i}\right)\left(\mathcal{Q}_{j}-e_{j}\right) X_{\mathcal{Q}_{i}}\left(X_{\mathcal{Q}_{j}} I\right)+ \\
(3.17) \frac{1}{3 !} \sum_{i, j, k=1}^{4}\left(\mathcal{Q}_{i}-e_{i}\right)\left(\mathcal{Q}_{j}-e_{j}\right)\left(\mathcal{Q}_{k}-e_{k}\right) X_{\mathcal{Q}_{i}}\left(X_{\mathcal{Q}_{j}}\left(X_{\mathcal{Q}_{k}} I\right)\right)+\cdots=0
\end{gathered}
$$

For every pair of values $M$ and $H$ equation (3.17) becomes a polynomial in the variables $\alpha, \beta, \gamma, \delta$. Consequently equation (3.17) can only hold if for every pair of values $M$ and $H$ the coefficients of the polynomial vanish, i.e., if the following relations hold

$$
\begin{aligned}
X_{\mathcal{Q}_{i}} I= & X_{\mathcal{Q}_{i}}\left(X_{\mathcal{Q}_{j}} I\right)= \\
& X_{\mathcal{Q}_{i}}\left(X_{\mathcal{Q}_{j}}\left(X_{\mathcal{Q}_{k}} I\right)\right)=\cdots=0, \quad i, j, k \in\{1,2,3,4\},
\end{aligned}
$$


for every pair of values $M$ and $H$. If $X_{\mathcal{Q}_{i}} I=0, \quad \mathcal{Q}_{i}=\alpha, \beta, \gamma, \delta$, the rest of the relations (3.18) follow. Equations (3.16), $X_{\mathcal{Q}_{i}} I=0, \quad \mathcal{Q}_{i}=\alpha, \beta, \gamma, \delta$, are nothing but equations (3.2), (3.3), (3.4), and (3.5), respectively. This completes the proof.

Sophus Lie's great advance was to replace the complicated, nonlinear finite invariance condition (3.1) by the vastly more useful linear infinitesimal condition (3.16) and to recognize that if a function satisfies the infinitesimal condition then it also satisfies the finite condition, and vice versa, provided that the function is analytic in the parameters $\alpha, \beta, \gamma$, and $\delta$.

It is to be noted that in the proof of Lie's main Theorem (3.1) we used the following:

1. The assumption that cloud invariant $I$ is a function analytic in the parameters $\alpha, \beta, \gamma$, and $\delta$.

2. The assumption that the set of transformations $\mathcal{T}(A)$, and subsequently the set of transformations $A$, contain the identity element, which is obtained when $\alpha=1, \beta=0, \gamma=0$, and $\delta=1$.

3. The chain rule for the differentiation of composite functions.

Nowhere in the proof of Lie's main Theorem (3.1) is the assumption made that the set of transformations $\mathcal{T}(A)$ is closed under the usual composition of transformations, or equivalently, that the associated set of matrices $A$ is closed under the usual matrix multiplication. This will become evident and exemplified in subsection 5.2.1. where we find cloud invariants $I$ under a set of transformations $\mathcal{T}(A)$ which are such that the associated set of matrices $A$ are not closed under the usual multiplication of matrices.

\subsection{Cloud invariants in the general case}

As a first application of Theorem (3.1) we find the cloud invariants under a general matrix $A$. This is the content of the next Corollary.

Corollary 3.1. If a cloud of points is transformed via a matrix $A=\left(\begin{array}{cc}\alpha & \beta \\ \gamma & \delta\end{array}\right)$, then the only cloud invariants are:

1. The constant functions $I(M, H)=c, c \in R$.

2. The level curve $I(M, H)=0$ of the function $I(M, H)=\frac{H}{M^{2}}-1$.

Proof: According to Theorem (3.1) a function $I(\hat{M}, \hat{H})$, analytic in the parameters $\alpha, \beta, \gamma, \delta$, is a cloud invariant if and only if it satisfies the system of PDEs (3.2), (3.3), (3.4), and (3.5), which read: 


$$
\begin{aligned}
& \xi_{\alpha}^{1}(\mathbf{x}) \frac{\partial I}{\partial M}+\xi_{\alpha}^{2}(\mathbf{x}) \frac{\partial I}{\partial H}=-M \frac{\partial I}{\partial M}-2 H \frac{\partial I}{\partial H}=0 \\
& \xi_{\beta}^{1}(\mathbf{x}) \frac{\partial I}{\partial M}+\xi_{\beta}^{2}(\mathbf{x}) \frac{\partial I}{\partial H}=\left(H-2 M^{2}\right) \frac{\partial I}{\partial M}-2 H M \frac{\partial I}{\partial H}=0 \\
& \xi_{\gamma}^{1}(\mathbf{x}) \frac{\partial I}{\partial M}+\xi_{\gamma}^{2}(\mathbf{x}) \frac{\partial I}{\partial H}=\frac{\partial I}{\partial M}-2 M \frac{\partial I}{\partial H}=0 \\
& \xi_{\delta}^{1}(\mathbf{x}) \frac{\partial I}{\partial M}+\xi_{\delta}^{2}(\mathbf{x}) \frac{\partial I}{\partial H}=M \frac{\partial I}{\partial M}+2 H \frac{\partial I}{\partial H}=0 .
\end{aligned}
$$

We easily find that the only solutions to the last system of equations are:

1. The constant functions $I(M, H)=c, c \in R$.

2. The level curve $I(M, H)=0$ of the function $I(M, H)=\frac{H}{M^{2}}-1$.

This completes the proof.

The second invariant implies in particular that when the values of $\mathrm{M}$ and $\mathrm{H}$ are such that $H=M^{2}$, then their transformed values $\hat{H}$ and $\hat{M}$ are such that $\hat{H}=\hat{M}^{2}$.

\section{The One-Parameter Case}

The cloud invariants under a general matrix $A$, given in Corollary 3.1, do not lend themselves to practical implementation. This leads us to examining particular cases of $A$. We start by considering the one-parameter case, i.e. the case where the entries of the matrix $A$ are analytic functions of a single parameter $\varphi$. Interestingly enough it turns out that in this case we can find cloud invariants in closed form which can be practically implemented. The first step to prove this assertion is the next Corollary.

Corollary 4.1. Let $\left(x_{i}, y_{i}\right), i=1, \ldots, N$, be a cloud of points on the plane with linear coefficients $M$ and $H$, and let $\left(\hat{x}_{i}, \hat{y}_{i}\right), i=1, \ldots, N$, be the transformation of the cloud $\left(x_{i}, y_{i}\right), i=1, \ldots, N$, under a matrix $A(\varphi)=\left(\begin{array}{cc}\alpha(\varphi) & \beta(\varphi) \\ \gamma(\varphi) & \delta(\varphi)\end{array}\right)$, where $\alpha(\varphi), \beta(\varphi), \gamma(\varphi)$, and $\delta(\varphi)$, are real analytic functions of a parameter $\varphi \in \mathbf{R}$. We assume that there exists a value of $\varphi$, denoted by $\varphi^{*}$, such that $A\left(\varphi^{*}\right)=\mathbf{I}$, I the $2 \times 2$ identity matrix. An analytic function $I: \mathbf{R}^{2} \rightarrow \mathbf{R}$ is a cloud invariant if and only if the next equation holds:

(4.1) $\left[\left(H-2 M^{2}\right) \beta^{\prime}\left(\varphi^{*}\right)-\delta M+\gamma^{\prime}\left(\varphi^{*}\right)\right] \frac{\partial I}{\partial M}+2\left[\gamma^{\prime}\left(\varphi^{*}\right) M-\beta^{\prime}\left(\varphi^{*}\right) H M-\delta H\right] \frac{\partial I}{\partial H}=0$, where, $\delta=\alpha^{\prime}\left(\varphi^{*}\right)-\delta^{\prime}\left(\varphi^{*}\right)$. 
Proof: In order to find the cloud invariants we apply Theorem 3.1. The key point is that in the case under consideration all the entries of the matrix $A(\varphi)$ are functions of a single parameter $\varphi$. This implies in particular that equations (3.2), (3.3), (3.4), and (3.5), whose solution space are the cloud invariants, reduce to one equation:

$$
\begin{gathered}
\xi_{\varphi}^{1} \frac{\partial I}{\partial M}+\xi_{\varphi}^{2} \frac{\partial I}{\partial H}=0 \\
\xi_{\varphi}^{1}=\left(\frac{\partial \hat{M}}{\partial \varphi}\right)_{e}=\frac{\partial \mathcal{M}(M, H, 1,0,0,1)}{\partial \varphi}, \xi_{\varphi}^{2}=\left(\frac{\partial \hat{H}}{\partial \varphi}\right)_{e}=\frac{\partial \mathcal{H}(M, H, 1,0,0,1)}{\partial \varphi} .
\end{gathered}
$$

Differentiation is now with respect to $\varphi$, that is $Q=\varphi$. Consequently cloud invariants in the one-parameter case are solutions to equation (4.2) which reads:

$$
\left[\left(H-2 M^{2}\right) \beta^{\prime}\left(\varphi^{*}\right)-\delta M+\gamma^{\prime}\left(\varphi^{*}\right)\right] \frac{\partial I}{\partial M}+2\left[\gamma^{\prime}\left(\varphi^{*}\right) M-\beta^{\prime}\left(\varphi^{*}\right) H M-\delta H\right] \frac{\partial I}{\partial H}=0,
$$

where, $\delta=\alpha^{\prime}\left(\varphi^{*}\right)-\delta^{\prime}\left(\varphi^{*}\right)$. This completes the proof.

It is difficult to obtain in closed form the whole set of solutions of equation (4.1). However we can find, in closed form, a wide subclass of solutions of equation (4.1). This is the content of the following Theorem.

Theorem 4.1. A class of solutions of equation (4.1), and hence a family of invariants of a cloud of points $\left(x_{i}, y_{i}\right), i=1, \ldots, N$, when it is transformed under a matrix $A(\varphi)=\left(\begin{array}{ll}\alpha(\varphi) & \beta(\varphi) \\ \gamma(\varphi) & \delta(\varphi)\end{array}\right)$, is given by:

$$
I(M, H)=F\left(\frac{M^{2}-H}{\left(H \beta^{\prime}\left(\varphi^{*}\right)-\gamma^{\prime}\left(\varphi^{*}\right)+\delta M\right)^{2}}\right),
$$

where $F($.$) , is an arbitrary real valued function and \delta=\alpha^{\prime}\left(\varphi^{*}\right)-\delta^{\prime}\left(\varphi^{*}\right)$. We assume that $\alpha(\varphi), \beta(\varphi), \gamma(\varphi)$, and $\delta(\varphi)$, are real analytic functions of a single parameter $\varphi \in \mathbf{R}$. We also assume that there exists a value of $\varphi$, denoted by $\varphi^{*}$, such that $A\left(\varphi^{*}\right)=\mathbf{I}$, I being the $2 \times 2$ identity matrix.

Proof: In order to find solutions of equation (4.1) we use the undetermined coefficients method. This method consists in seeking for solutions $I(M, H)$ of the form:

$$
F\left(\frac{\sum_{i=1}^{n} \sum_{j=1}^{m} a_{i j} M^{i} H^{j}}{\sum_{i=1}^{n} \sum_{j=1}^{m} b_{i j} M^{i} H^{j}}\right),
$$

where $a_{i j}$ and $b_{i j}$ are unknown coefficients to be determined. By substituting this particular form of the solution into equation (4.1) we obtain that a polynomial in the two variables $M$ and $H$ is equal to zero. The resulting condition, the coefficients of the polynomial are equal to zero, gives solution (4.3). This completes the proof.

A Corollary of the previous theorem is that we can find cloud invariants, when the cloud is transformed under a matrix, provided the matrix is an element of the one-parameter set of transformations $A(\varphi)$, considered in this theorem. 
Corollary 4.2. Let $\left(x_{i}, y_{i}\right), i=1, \ldots, N$, be a cloud of points and let this cloud be transformed under a matrix $A=\left(\begin{array}{ll}a_{11} & a_{12} \\ a_{21} & a_{22}\end{array}\right)$, aij $\in \mathbf{R}$. If there exist real valued, analytic, functions $\alpha(\varphi), \beta(\varphi), \gamma(\varphi), \delta(\varphi)$ and values $\varphi^{*}, \varphi_{1}$, such that:

1. $\alpha\left(\varphi^{*}\right)=1, \beta\left(\varphi^{*}\right)=0, \gamma\left(\varphi^{*}\right)=0, \delta\left(\varphi^{*}\right)=1$

2. $\alpha\left(\varphi_{1}\right)=a_{11}, \beta\left(\varphi_{1}\right)=a_{12}, \gamma\left(\varphi_{1}\right)=a_{21}, \delta\left(\varphi_{1}\right)=a_{22}$

then, the quantity

$$
I(M, H)=F\left(\frac{M^{2}-H}{\left(H \beta^{\prime}\left(\varphi^{*}\right)-\gamma^{\prime}\left(\varphi^{*}\right)+\delta M\right)^{2}}\right),
$$

where $F($.$) is an arbitrary real valued function and \delta=\alpha^{\prime}\left(\varphi^{*}\right)-\delta^{\prime}\left(\varphi^{*}\right)$, is a cloud invariant.

Proof: This is an immediate consequence of Theorem 4.1.

\section{Examples of Invariants}

In this section, by using Theorem theorem 4.1, we find cloud invariants when a cloud is transformed under various sets of transformations $A(\varphi)$. As we pointed out in section 2 . it is not necessary the set $A(\varphi)$ to form a group under the usual multiplication of matrices. Firstly we consider sets of transformations $A(\varphi)$ which do form a group and then we consider a set $A(\varphi)$ which does not form a group. Finally, in the last subsection, by using the previous findings, we find cloud invariants for any given matrix.

\subsection{Sets $A(\varphi)$ which form a group}

We start with simpler sets of transformations $A(\varphi)$ and gradually proceed to more general cases.

\subsection{1. $A(\varphi)$ is a diagonal matrix}

We start by assuming that $A(\varphi)$ is diagonal and has the form:

$$
A(\varphi)=\left(\begin{array}{ll}
1 & 0 \\
0 & \varphi
\end{array}\right)
$$

where $\varphi \in \mathbf{R}$. 
In this case we can easily see that $\varphi^{*}=1$ and that $\beta^{\prime}\left(\varphi^{*}\right)=0, \gamma^{\prime}\left(\varphi^{*}\right)=$ $0, \delta=\alpha^{\prime}\left(\varphi^{*}\right)-\delta^{\prime}\left(\varphi^{*}\right)=-1$. Therefore, according to Theorem 4.1, a family of cloud invariants is:

$$
F\left(\frac{M^{2}-H}{(-M)^{2}}\right)=h\left(\frac{H}{M^{2}}\right),
$$

where $F(\cdot)$ and $h(\cdot)$ are arbitrary real valued functions.

\subsection{2. $A(\varphi)$ is an upper triangular matrix}

We assume that $A(\varphi)$ is upper triangular and has the form:

$$
A(\varphi)=\left(\begin{array}{ll}
1 & \varphi \\
0 & 1
\end{array}\right)
$$

$\varphi \in \mathbf{R}$.

In this case we easily verify that $\varphi^{*}=0$ and that $\beta^{\prime}\left(\varphi^{*}\right)=1, \gamma^{\prime}\left(\varphi^{*}\right)=0, \delta=$ $\alpha^{\prime}\left(\varphi^{*}\right)-\delta^{\prime}\left(\varphi^{*}\right)=0$. Consequently, according to Theorem 4.1, a family of cloud invariants is:

$$
F\left(\frac{M^{2}-H}{(H \cdot 1)^{2}}\right)=F\left(\frac{M^{2}-H}{H^{2}}\right),
$$

where $F(\cdot)$ is an arbitrary real valued function.

\subsection{3. $A(\varphi)$ is a lower triangular matrix}

We assume that $A(\varphi)$ is lower triangular and has the form:

$$
A(\varphi)=\left(\begin{array}{ll}
1 & 0 \\
\varphi & 1
\end{array}\right)
$$

$\varphi \in \mathbf{R}$

In this case we easily find that $\varphi^{*}=0$ and that $\beta^{\prime}\left(\varphi^{*}\right)=0, \gamma^{\prime}\left(\varphi^{*}\right)=1, \delta=$ $\alpha^{\prime}\left(\varphi^{*}\right)-\delta^{\prime}\left(\varphi^{*}\right)=0$. Consequently, according to Theorem 4.1, a family of cloud invariants is:

$$
F\left(\frac{M^{2}-H}{(-1)^{2}}\right)=h\left(H-M^{2}\right),
$$

where $F(\cdot)$ and $h(\cdot)$ are arbitrary real valued functions.

\subsection{4. $A(\varphi)$ is a rotation matrix}


Finally, we assume that $A(\varphi)$ is a rotation matrix and has the form:

$$
A(\varphi)=\left(\begin{array}{cc}
\cos \varphi & \sin \varphi \\
-\sin \varphi & \cos \varphi
\end{array}\right)
$$

$\varphi \in \mathbf{R}$.

In this case we easily obtain that $\varphi^{*}=0$ and that $\beta^{\prime}\left(\varphi^{*}\right)=1, \gamma^{\prime}\left(\varphi^{*}\right)=-1, \delta=$ $\alpha^{\prime}\left(\varphi^{*}\right)-\delta^{\prime}\left(\varphi^{*}\right)=0$. Consequently, according to Theorem 4.1, a family of cloud invariants is:

$$
F\left(\frac{M^{2}-H}{(H+1)^{2}}\right)
$$

where $F(\cdot)$ is an arbitrary real valued function.

\subsection{A set $A(\varphi)$ which does not form a group}

\subsubsection{A "linear" matrix}

A set of transformations $A(\varphi)$ which subsumes the sets of transformations considered in subsections 5.1.1., 5.1.2., and 5.1.3. is the set of "linear" matrices

$$
A(\varphi)=\left(\begin{array}{ll}
a_{11} & a_{12} \\
a_{21} & a_{22}
\end{array}\right)+\varphi\left(\begin{array}{ll}
b_{11} & b_{12} \\
b_{21} & b_{22}
\end{array}\right)=\left(\begin{array}{cc}
a_{11}+b_{11} \varphi & a_{12}+b_{12} \varphi \\
a_{21}+b_{21} \varphi & a_{22}+b_{22} \varphi
\end{array}\right),
$$

where $a_{i j}, b_{i j} \in \mathbf{R}$, and $\varphi$ is a real free parameter. The set of matrices $A\left(\varphi^{*}\right)$ does not, in general, form a group under matrix multiplication. However, we assume that there exists a value of $\varphi$, denoted by $\varphi^{*}$, such that $A\left(\varphi^{*}\right)=\mathbf{I}, \mathbf{I}$ being the $2 \times 2$ identity matrix. One can easily check that such a value $\varphi^{*}$ exists if and only if the entries $a_{i j}, b_{i j}$ satisfy one of the following conditions:

$$
b_{22} \neq 0 \wedge a_{21}=\frac{\left(a_{22}-1\right) b_{21}}{b_{22}} \wedge a_{12}=\frac{\left(a_{22}-1\right) b_{12}}{b_{22}} \wedge a_{11}=\frac{a_{22} b_{11}-b_{11}+b_{22}}{b_{22}}
$$

$$
b_{22}=0 \wedge a_{22}=1 \wedge b_{21} \neq 0 \wedge a_{12}=\frac{a_{21} b_{12}}{b_{21}} \wedge a_{11}=\frac{a_{21} b_{11}+b_{21}}{b_{21}},
$$

$$
b_{22}=0 \wedge b_{21}=0 \wedge a_{22}=1 \wedge a_{21}=0 \wedge b_{12} \neq 0 \wedge a_{11}=\frac{a_{12} b_{11}+b_{12}}{b_{12}}
$$

(5.13) $\quad b_{22}=0 \wedge b_{21}=0 \wedge b_{12}=0 \wedge a_{22}=1 \wedge a_{21}=0 \wedge a_{12}=0 \wedge b_{11} \neq 0$.

In this case we easily find that $\beta^{\prime}\left(\varphi^{*}\right)=b_{12}, \gamma^{\prime}\left(\varphi^{*}\right)=b_{21}, \delta=\alpha^{\prime}\left(\varphi^{*}\right)-\delta^{\prime}\left(\varphi^{*}\right)=$ $b_{11}-b_{22}$. According to Theorem 4.1, a family of cloud invariants is:

$$
F\left(\frac{M^{2}-H}{\left(H b_{12}-b_{21}+\left(b_{11}-b_{22}\right) M\right)^{2}}\right),
$$

where $F(\cdot)$ is an arbitrary real valued function. 


\subsubsection{Cloud invariants for an arbitrary matrix}

For any given matrix there always exists a set of transformations $A(\varphi)$, of the form (5.9), which contains this matrix. In fact one can easily prove that there exists a two parameter family of such sets $A(\varphi)$. According to Corollary 4.2, a family of cloud invariants, when a cloud of points is transformed under this matrix, is given by relation (5.14). As a case study we consider the matrix:

$$
\mathcal{M}=\left(\begin{array}{cc}
0.4 & -0.4 \\
-0.05 & 0.9
\end{array}\right)
$$

Let $A(\varphi)$ be the set of transformations:

$$
A(\varphi)=\left(\begin{array}{cc}
-2 & -2 \\
-1 / 4 & 1 / 2
\end{array}\right)+\varphi\left(\begin{array}{cc}
12 & 8 \\
1 & 2
\end{array}\right)=\left(\begin{array}{cc}
-2+12 \varphi & -2+8 \varphi \\
-1 / 4+\varphi & 1 / 2+2 \varphi
\end{array}\right)
$$

One can easily check that $A(0.2)=\mathcal{M}$. We have $b_{12}=8, b_{21}=1, b_{11}-b_{22}=10$. According to Corollary 4.2, a family of cloud invariants is:

$$
F\left(\frac{M^{2}-H}{(8 H-1+10 M)^{2}}\right),
$$

where $F(\cdot)$ is an arbitrary real valued function. Since there exists a two parameter family of sets $A(\varphi)$, of the form (5.9), which contain $\mathcal{M}$, there exists a two parameter family of cloud invariants of the form (5.17), when a cloud is transformed via $\mathcal{M}$. However, the explicit form of this two parameter family of cloud invariants is not needed here.

\section{Simulations}

To see how the above theory works in practise, we consider a cloud of 10.000 points forming the scheme of Figure 6.1. Using relations (2.1) and (2.2), we calculate the linear coefficients $M$ and $H$ of the cloud. We find $M=1.52244$ and $H=$ 2.46998. We transform now this cloud by using various matrices.

Firstly we let the the diagonal matrix $A=\left(\begin{array}{ll}1 & 0 \\ 0 & 2\end{array}\right)$ to act on the cloud. Both the initial and the transformed schemes are depicted in Figure 6.2. Using relations (2.3) and (2.4) we calculate the linear coefficients of the new cloud and we obtain $\hat{M}=3.04488$ and $\hat{H}=9.87991$. According to our findings in subsection 5.1.1., a family of cloud invariants, when a cloud is transformed with the diagonal matrix $A$, is $I(M, H)=F\left(H / M^{2}\right)$, where $F(\cdot)$ is an arbitrary real valued function. Indeed, for the initial and the transformed linear coefficients, we find $H / M^{2}=\hat{H} / \hat{M}^{2}=$ 1.06564. It follows that we have $I(M, H)=I(\hat{M}, \hat{H})$ for any real valued function $F(\cdot)$. 


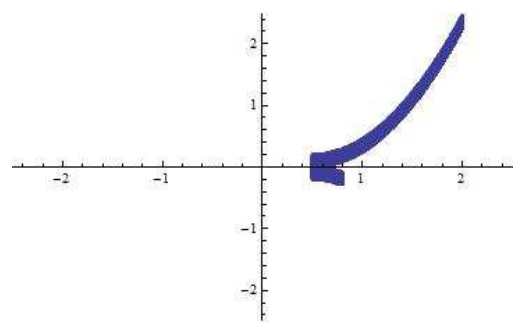

FIG. 6.1: The Original Scheme

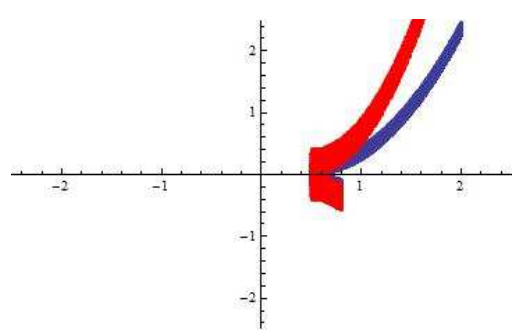

Fig. 6.2: A Diagonal Transformation

As a second example of transformation, we let the upper triangular matrix $B=$ $\left(\begin{array}{cc}1 & 0.7 \\ 0 & 1\end{array}\right)$ to act on the cloud. The result of this transformation is given in Figure 6.3. The transformation of the cloud we consider under $B$ has linear coefficients $\hat{M}=0.748882$ and $\hat{H}=0.568896$. As we found in subsection 5.1.2., a family of cloud invariants, when a cloud is transformed with the upper triangular matrix $B$, is $I(M, H)=F\left(\left(M^{2}-H\right) / H^{2}\right)$, where $F(\cdot)$ is an arbitrary real valued function. Indeed, we have $\left(M^{2}-H\right) / H^{2}=\left(\hat{M}^{2}-\hat{H}\right) / \hat{H}^{2}=-0.0249396$. Consequently we have $I(M, H)=I(\hat{M}, \hat{H})$ for any real valued function $F(\cdot)$.

As a third example of transformation, we act on the cloud of points with a rota-

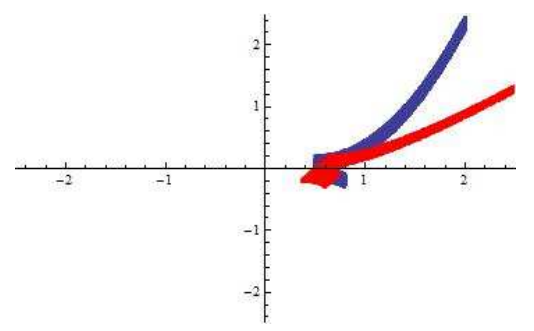

FIG. 6.3: An Upper Triangular Transformation 


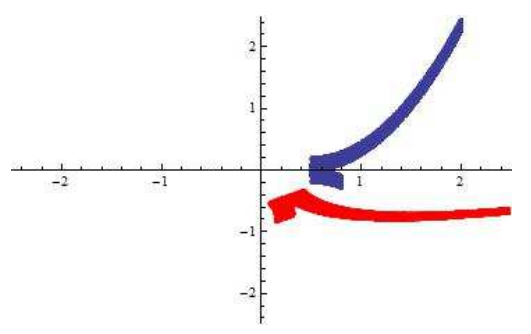

FIG. 6.4: A Rotation

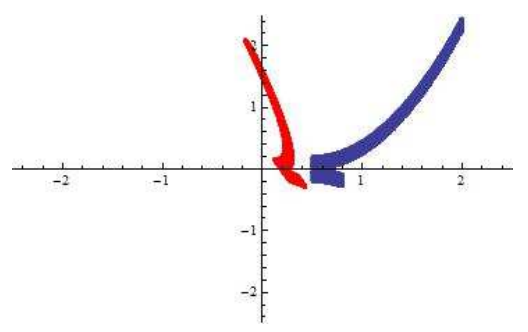

FIG. 6.5: An Arbritrary Matrix

tion matrix $C=\left(\begin{array}{cc}\cos \frac{\pi}{3} & \sin \frac{\pi}{3} \\ -\sin \frac{\pi}{3} & \cos \frac{\pi}{3}\end{array}\right)$. The initial and the rotated clouds are shown

in Figure 6.4. The linear coefficients of the rotated cloud are $\hat{M}=-0.0364518$ and $\hat{H}=0.0143303$. We found in subsection 5.1.4., that a family of cloud invariants, when a cloud is transformed with the rotation matrix $C$, is $I(M, H)=$ $F\left(\frac{M^{2}-H}{(H+1)^{2}}\right)$, where $F(\cdot)$ is an arbitrary real valued function. Indeed, we have $\frac{M^{2}-H}{(H+1)^{2}}=\frac{\hat{M}^{2}-\hat{H}}{(\hat{H}+1)^{2}}=-0.0126368$. Henceforth we have $I(M, H)=I(\hat{M}, \hat{H})$, for any real valued function $F(\cdot)$.

Finally, we act on the cloud of points with the matrix $D=\left(\begin{array}{cc}0.4 & -0.4 \\ -0.05 & 0.9\end{array}\right)$. The result of this transformation is shown in Figure 6.5. The linear coefficients of the transformed cloud are $\hat{M}=-4.86159$ and $\hat{H}=27.4371$. We found in subsection 5.2.1., that a family of cloud invariants, when a cloud is transformed with the matrix $D$, is $I(M, H)=F\left(\frac{M^{2}-H}{(8 H-1+10 M)^{2}}\right)$, where $F(\cdot)$ is an arbitrary real valued function. Indeed, we have $\frac{M^{2}-H}{(8 H-1+10 M)^{2}}=\frac{\hat{M}^{2}-\hat{H}}{(8 \hat{H}-1+10 \hat{M})^{2}}=-0.000131743$. Consequently we obtain $I(M, H)=I(\hat{M}, \hat{H})$, for any real valued function $F(\cdot)$. 
We note that the results we obtained by considering the aforementioned cloud of points verify our findings in section 5 .

\section{Concluding Remarks}

We have studied transformations, with $2 \times 2$ matrices $\left(\begin{array}{cc}\alpha & \beta \\ \gamma & \delta\end{array}\right), \alpha, \beta, \gamma, \delta \in \mathbf{R}$, of planar set of points, called clouds of points for convenience. Our aim in this paper is to find cloud invariants, i.e. functions which take the same value when they are evaluated for the initial and for the transformed cloud of points. It is natural the cloud invariants to be functions of variables which carry information for the whole cloud. The cloud invariants we find are functions of two such variables $M$ and $H$.

$M$ and $H$ are functions of the coordinates of the points of the cloud. As a result we find that any transformation of a cloud of points by a $2 \times 2$ matrix induces a nonlinear transformation $(M, H) \rightarrow(\hat{M}, \hat{H}), \hat{M}=\mathcal{M}(M, H, \alpha, \beta, \gamma, \delta), \hat{H}=$ $\mathcal{H}(M, H, \alpha, \beta, \gamma, \delta)$, given explicitly by equations (2.3) and (2.4), of the variables $M$ and $H$.

$M$ and $H$ originate from the best fitting straight line through the cloud of points under consideration. This straight line is determined by the least squares fitting technique. Henceforth by definition a cloud invariant is any function $I(M, H)$ which satisfies the relation (3.1), I(M,H) $I(\hat{M}, \hat{H})$, where $\hat{M}$ and $\hat{H}$ are the values of the variables $M$ and $H$ for the transformed cloud.

We find cloud invariants by using Lie theory. Lie theory replaces the complicated, nonlinear finite invariance condition (3.1) by the more useful and tractable linear infinitesimal condition (3.16) provided that the function $I(\hat{M}, \hat{H})$ is analytic in the parameters $\alpha, \beta, \gamma$, and $\delta$. Linear condition (3.16) is a set of linear PDEs. Any solution to this system of PDEs gives a cloud invariant.

Cloud invariants can be practically implemented in various fields, e.g. in optical character recognition, in image analysis and computer graphics techniques, by providing the necessary tools in order to identify icons created by the same "source". The cloud invariants we find for the general four-parameter case, when a cloud is transformed with a matrix $\left(\begin{array}{cc}\alpha & \beta \\ \gamma & \delta\end{array}\right)$, cannot be practically implemented.

However, the cloud invariants we find for various one-parameter groups of transformations can be practically implemented. In particular we find cloud invariants for a group consisting of diagonal matrices, for a group consisting of upper triangular matrices, for a group consisting of lower triangular matrices, and for the group of rotations $S O(2)$.

More importantly, for the practical implementation of our findings, we find cloud invariants for any given matrix. We find these cloud invariants by noticing that any given matrix belongs to a one-parameter "linear" set of transformations of the form $\mathcal{A}+\mathcal{B} \varphi, \mathcal{A}$ and $\mathcal{B}$ are given $2 \times 2$ matrices, and $\varphi \in \mathbf{R}$. Our findings are verified by examples and simulations in a cloud of 10.000 points. 
We expressed the cloud invariants in terms of the variables $M$ and $H . M$ and $H$ are essentially the coefficients of the straight line which is the best fit for the cloud of points under consideration. This provides a natural guide for future research. With a view to apply our results in fields such as character recognition, the next logical step is to consider the case where the cloud of points originates from an icon which has a parabolic-like shape.

In this case we will look for cloud invariants which are expressed in terms of variables which appear as coefficients, or variations thereof, of the parabola which is the best fit for the cloud of points under consideration. For similar reasons, subsequently, we will look for new cloud invariants expressed in terms of variables which appear as coefficients in third or higher degree curves. We will compare our findings, with simulations and computational experiments, with those acquired by other approaches.

\section{Acknowledgement}

The first author would like to express his thanks to Mr. Koutsoulis Nikos, for his attempts to face the problem initially.

\section{R E F E R E N C E S}

1. B. J. CANTWELL: Introduction to Symmetry Analysis. Cambridge University Press, New York, 2002.

2. J. FLUSSER: Moment Invariants in Image Analysis. International Journal of Computer, Electrical, Automation, Control and Information Engineering 1 (2007), N.11

3. J. FLUSSER and B. ZITOVA: Combined Invariants to Convolution and Rotation and their Application to Image Registration. Advances in Pattern Recognition ICAPR 2001, V. 2013 of the Series Lecture Notes in Computer Science, 361-370.

4. J. FLUSSER and T. SUK: Degraded image analysis: An invariant approach. IEEE Transactions on Pattern Analysis and Machine Intelligence, 20 N.6 (1998), 590-603.

5. R. GILMORE: Lie Groups, Physics, and Geometry. Cambridge University Press, New York, 2008.

6. M. K. HU: Visual Pattern Recognition by Moment Invariants. IRE Transactions of Information Theory. 8 (1962), 179-187.

7. N. KALOUPTSIDIS: Signal Processing Systems, Theory and Design. John Wiley and Sons, 1997.

8. M. D. NEUSEL: Invariant Theory. (Student Mathematical Library. Band 36) American Mathematical Society, Providence R. I., 2007.

9. J. M. NILOY: Estimating surface normals in noisy point cloud data, SoCG'03 Proceedings of the nineteenth annual symposium on Computational geometry, ACM, New York, 2003, pp. 322-328.

10. H. SCHWEITZER and J. STRAACH: Utilizing Moment Invariants and Groebner Bases to Reason About Shapes. Computational Intelligence 14 (1998), 461-474. 
Stelios Kotsios

University of Athens

Department of Economics

Unit of Mathematics and Informatics

Sofokleous 1

10559 Athens, Greece

skotsios@econ.uoa.gr

Evangelos Melas

University of Athens

Department of Economics

Unit of Mathematics and Informatics

Sofokleous 1

10559 Athens, Greece

emelas@econ.uoa.gr 